\title{
Telehealth for people with spinal cord injury: a narrative review
}

\author{
I Irgens ${ }^{1} \cdot \mathrm{T}$ Rekand $\mathbb{D}^{2,3} \cdot \mathrm{M}$ Arora $\mathbb{C}^{4,5} \cdot \mathrm{N} \mathrm{Liu^{6 }} \cdot \mathrm{R}$ Marshall ${ }^{7,8} \cdot \mathrm{F}$ Biering- Sørensen ${ }^{9} \cdot \mathrm{M}$ Alexander ${ }^{10,11,12}$
}

Received: 8 July 2017 / Revised: 30 September 2017 / Accepted: 9 October 2017 / Published online: 7 March 2018

(c) The Author(s) 2018. This article is published with open access

\begin{abstract}
Study design Narrative review.

Objectives To find and discuss what has been published about the use of telehealth, on people with spinal cord injury (teleSCI).

Setting International.

Method Cochrane Library, Ovid Medline, EMBASE and CINAHL, from 1996 till June 2017 have been searched. Searches in PsycINFO, from 1996 till September 2017, were included afterwards. Extracted data include studies in English language, containing information about spinal cord injury and disorders, and telehealth. Literature reviews, systematic reviews, and studies containing other types of neurological disorders, were excluded. Studies were grouped based on how and to whom telehealth was offered.

Results Twenty nine studies were included in the review. They were categorized according to the way teleSCI was provided, and to what modality was used. Some studies utilized more than one modality. TeleSCI seems to be favorable concerning treatment and follow-up, as well as favorable socioeconomically and environmentally. The studies spanned across several aims and outcomes. There was also heterogeneity in number of participants, the differences in modalities, and in the level of evidence. Thus it was challenging to compare studies and make future recommendations.

Conclusions TeleSCI can be used for examination and guiding purposes. Further research is warranted to evaluate optimal utilization, methodology and efficacy.

Sponsorship The first author has received funding from the Norwegian Extra Foundation.
\end{abstract}

Electronic supplementary material The online version of this article (https://doi.org/10.1038/s41393-017-0033-3) contains supplementary material, which is available to authorized users.

\section{Alexander}

spinalcordmd@live.com

1 Research Department, Sunnaas Rehabilitation Hospital, Norway, University of Oslo, Oslo, Norway

2 Department of Neurology, Haukeland University Hospital, Bergen, Norway

3 Sahlgrenska Academy, Institute for Physiology and Neuroscience, University of Gothenburg, Gothenburg, Sweden

4 John Walsh Centre for Rehabilitation Research, Kolling Institute of Medical Research, Northern Sydney Local Health District, Sydney, St Leonards, New South Wales 2065, Australia

5 Sydney Medical School Northern, The University of Sydney, Sydney, New South Wales 2006, Australia

6 Department of Rehabilitation Medicine, Peking University Third Hospital, Beijing, China

\section{Introduction}

One of the most significant changes in the practice of medicine has been the use of telemedicine [1]. Telemedicine can be defined as "the investigation, monitoring and

7 South Australian Spinal Cord Injury Service, Central Adelaide Local Health Network, Hampstead Rehabilitation Centre,

Lightsview, Adelaide, SA, Australia

8 Discipline of Orthopaedics and Trauma, School of Medicine, Faculty of Health Sciences, University of Adelaide, Adelaide, SA, Australia

9 Clinic for Spinal Cord Injuries, Rigshospitalet, University of Copenhagen, Copenhagen, Denmark

10 Birmingham Veterans Affairs Medical Center, Birmingham, Birmingham, Alabama, USA

11 Department of Physical Medicine and Rehabilitation, University of Alabama at Birmingham School of Medicine, Birmingham, AL, USA

12 Spaulding Rehabilitation Hospital, Boston, Massachusetts, USA 
management of patients and education of patients and staff, using systems which allow access to expert advice and patient information, no matter where the patient or relevant information is located [2]". Telemedicine was used as early as in the 1930s for telephone consultations for passengers on the Queen Mary [3]. Recently, the use of telemedicine has expanded rapidly with services available directly to patient for common concerns, such as upper respiratory and urinary tract infections [4] and through consultation from healthcare facilities for issues such as diabetic retinopathy [5], acute stroke [6] and intensive care unit patient management [7].

People with spinal cord injury (SCI) have difficulties with transportation and they represent a group that could benefit from using telemedicine services [8]. Moreover, there are few specialists who are qualified to care for persons with SCI/D and patients often reside far from their providers. At the International Spinal Cord Society (ISCoS) Annual Scientific Meeting in Vienna, Austria on 16 September 2016 [9], an international panel of leaders in telehealth services put on an instructional course regarding telemedicine and SCI/D, coining the term "telespinalcordinjury", or teleSCI. Subsequent to this forum, we determined a need to describe what has been published about this topic with the aim to find what is the status of the use of telehealth in the group of people with SCI. We chose to limit our search to only apply to this group, because of the profound mobility impairments associated with SCI as compared to other disabilities.

\section{Method}

Articles included in this narrative review $[10,11]$ are listed in Table 1.

\section{Searches}

Searches on the databases (The Cochrane Library, Ovid Medline, EMBASE, and CINAHL) were set to a time frame of 1996 to June 2017. These searches were carried out on October 15, 2016, November 16, 2016 and finally on June 6, 2017. PsycINFO was added with a search on September 5, 2017. Searches were created in collaboration with a Senior Librarian with expertise in medical science searches, working at the University Library of Oslo. Our search strategies included primary and secondary search terms in both medical subject headings (MeSH) and in title/abstract. Filter terms (year from 1996-current; English and Scandinavian language) were used to eliminate irrelevant articles (supplementary materials 1-2). We limited our search to the 1996-2017 period, because we wanted to include the use of primarily new telehealth technology. The English language was chosen as filter term, supplemented by the Scandinavian languages, because three of the authors are familiar with these languages. No articles about the topic were found in any of the Scandinavian languages. The Ovid Medline strategy was adapted to the syntax and subject headings of the other databases (Supplementary materials 3).

\section{Eligibility criteria}

We included randomized controlled trials (RCTs), nonrandomized studies, case series with more than five participants and interrupted time serial studies with more than ten participants, both genders and all ages. We excluded studies not in English or Scandinavian languages, literature reviews, systematic reviews, and studies containing a majority of individuals with other neurological disorders.

\section{Categorization of articles}

Only original research articles were included (Supplementary materials 4-7). Titles were reviewed first, followed by assessing relevant abstracts. Subsequently inclusion and exclusion criteria were utilized to determine which papers were selected for full reading and potential review. The results were divided into groups focusing on SCI and telehealth (teleSCI), with attention to how the teleSCI support was provided, leading to four different groups; voice only (telephone or mechanical voice), video plus voice (screen/ videoconferencing), computer only (laptop/PC/tablets) and robotic voice or video (mechanical voice and/or animation). We then focused on to whom the telehealth service was given, and divided the results in five different groups, containing provider to provider (health care workers to health care workers or to other caregivers), direct to consumer (health care workers to patients), store and forward (transmission of information to health care receivers or users, not active live communication), web-based treatments (online education) and interactive home monitoring (e.g. with home based vital signs monitors) (Table 2). For purposes of our discussion, we use the term "direct to consumer" when care is included as one to one treatment to the home.

\section{Outcome measures}

TeleSCI plus standard care was compared to standard care alone, to evaluate the benefit of teleSCI. Standard treatment included general outpatient treatment and inpatient treatment. We included adverse effects in the grading, when presented in the studies. All effects are mentioned as presented in the studies along with a short explanation/definition for each, with the aim of documenting the overall effect of teleSCI. The degree various forms of teleSCI have been shown to benefit patients in psychosocial (mental health, 
Fig. 1 Flowchart of the selection of articles

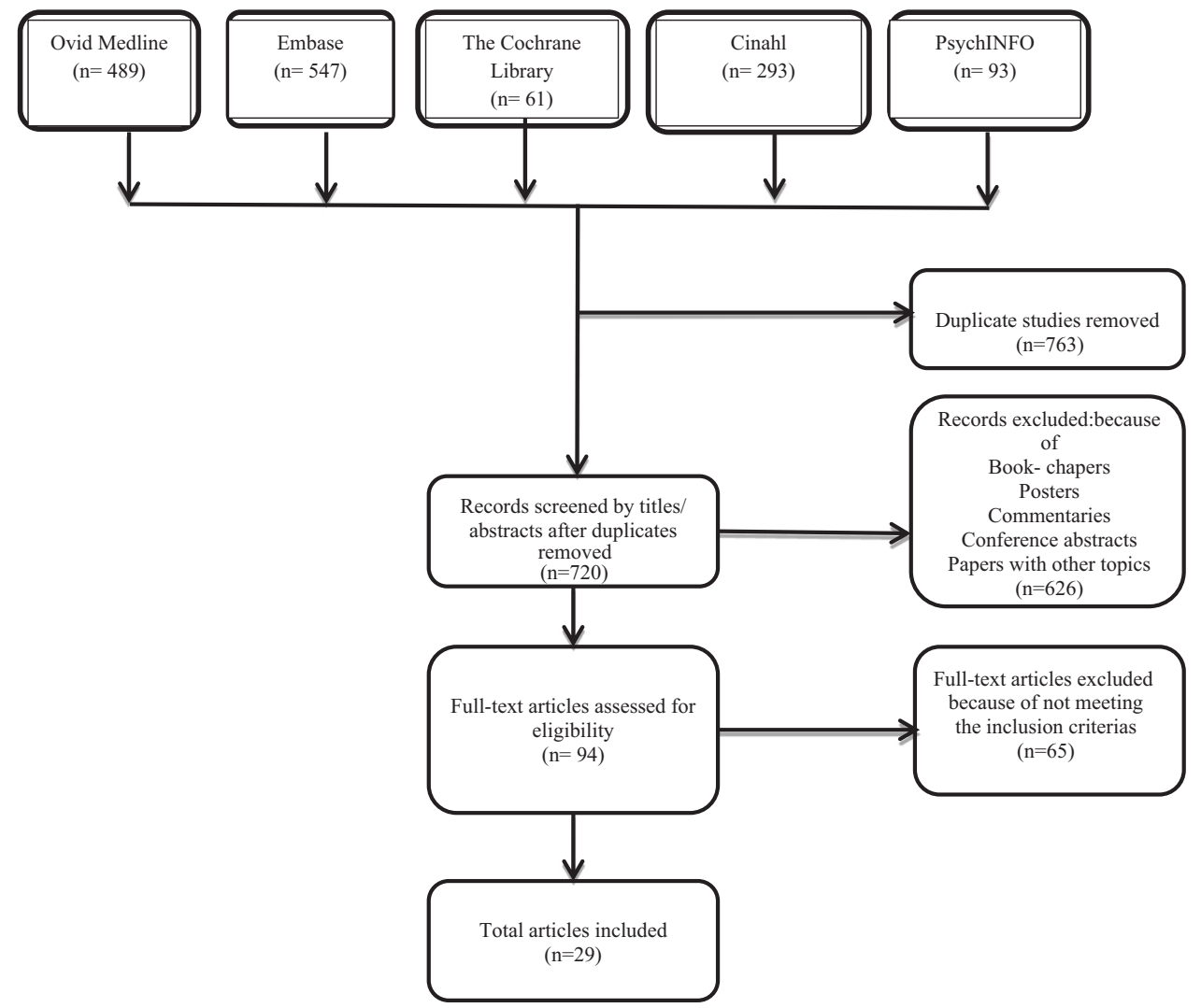

family, vocational counseling), medical (e.g., pressure sores, upper respiratory infections, spasticity), and functional (e.g., physical, occupational and recreational therapies) ways, are documented. Satisfaction, time and money saved, are reported when available (Table 1).

\section{Selection of studies}

The first author (II) conducted the initial search together with the librarian. The first author then chose the selection of studies for further investigation. The selection was double-checked by the last author (MAl). The librarian entered each selected article into a reference management database and duplicates were excluded (Fig. 1). Three groups of authors, two authors in each group (II and TR, NL and F B-S, MAr and RM), extracted information from the articles into a summary table (Table 1). Any disagreement in the groups was resolved by the last author (MAl). Each article that met the inclusion criteria is summarized in this table. Abstracted data included categories and modalities of the teleSCI used, diagnostic information, methodology, study design details, number of included/excluded individuals, number of individual responders, feasibility and outcome measures of the study (Table 1).

\section{Data analysis}

Following data extraction, study characteristics and variables were tabulated. Because of the small number of articles, the large heterogeneity in the study types, and because it was not possible to make a well-defined review question, both a formal meta-analysis and a structured review were deemed not eligible, and a narrative review was performed.

\section{Results}

A total of 1483 potentially relevant articles were identified (Supplementary materials $4-7)$. Duplicates $(n=763)$ were removed. Title and abstract screening identified 94 articles meeting the inclusion criteria for the abstract review, and the full text of the articles read. Twenty nine full text articles met the inclusion criteria for the review. Sixty five studies were excluded. A summary is provided in the flow diagram (Fig. 1). Detailed study results are presented in Table 1.

Based on the categories we found two studies considered provider to provider, 19 direct to consumer, one store and forward, six web-based treatments and three interactive home monitoring studies. Thirteen of the studies included the modality voice only, nine video plus voice, seven computer, and two robotic voice or video. In some of the 
categories, teleSCI care was provided via multiple modalities (Table 2).

\section{Provider to provider}

Provider to provider treatment is used for collaboration between different hospital departments or between specialized health care and municipality. One retrospective study was classified as provider to provider [12]. In this study the authors looked at a convenience sample of veterans in the United States (US) with diagnosis of pressure ulcers (PUs), and then compared the costs of those veterans who had been treated with teleSCI, vs. those who had not been treated with teleSCI through the Veteran Affairs (VA) Decision Support System. Using this methodology, costs of providing specialty wound care to 76 SCI veterans by video plus voice teleconsultation vs. traditional care, in terms of inpatient admissions, bed days of care, outpatient visits, overall cost for outpatient care and overall cost for inpatient skin/ wound care were compared. The teleSCI group had more outpatient encounters (median 12 vs. $4, p=0.007$ ) and longer inpatient stays, compared to traditional care (81 vs. 19 days/admission). The median cost per outpatient encounter was higher ( $\$ 440$ vs. $\$ 141, p<0.0001)$ in the teleSCI group. There were no significant differences in inpatient admission cost. This study retrospectively compared two groups-one treated with telemedicine, and we do not know the reasons why telemedicine was chosen for the individuals, or if all necessary information was loaded into the VA Decision Support System. We do not know the distance between the subjects and the facilities they received care in, the support systems or the degrees of PUs in the two groups, thus these results cannot be generalized.

Although not traditionally provider to provider, Careau [13], assessed the feasibility of video-conferencing for the inter-professional team managing people with SCI. Video conferencing was used to incorporate healthcare workers, community partners, patients and their caregivers as part of team meetings. Themes were assessed in 11 video conferencing meetings involving 47 healthcare workers, five community partners, eight patients and their five relatives. A typical videoconference involved 12 participants with average duration of $48 \mathrm{~min}$. The authors noted a high rate of productivity $(90.6 \%)$ along with very little time focused on resolving conflicts $(5.5 \%)$ and discussion of unrelated topics $(4.0 \%)$. "Significant changes of the client" were most often addressed (18.4\%).

\section{Direct to consumer}

As a reference for whether teleSCI therapy is feasible, it is important to know what percentage of the SCI population actively engages in use of computer technology.
Hogan et al. [14] studied 290 US veterans. With a $38 \%$ response rate, findings revealed $64.8 \%$ of veterans used a PC, $62.9 \%$ used the Internet, and $26.2 \%$ used text messaging. In another article [34], the interrater reliability of administration of the SCI secondary condition scale by telephone, was tested in 40 individuals with SCI. Strong interrater reliability was noted with a paper version of the instrument. Another group [15] documented patient satisfaction in 29 persons who had received a teleconsultation after rehabilitation discharge. Thus, the use of direct to consumer teleSCI seems feasible. This is also what Houlihan et al. [16] found in their study, evaluating the impact of "My Care My Call" (MCMC) — a peer-led, telephone-based empowerment intervention. The authors concluded that "the MCMC peer-led, health self-management intervention achieved a positive impact on self-management to prevent secondary conditions in adults with SCI".

A single-site, single-blinded RCT evaluating 168 individuals receiving usual care plus telephone counseling vs. usual care alone [17] evaluated self-reported health care utilization and medical complications, depression severity, current health state, subjective health and community participation in the US. The groups were similar at baseline, except that more control participants were married. There were no differences between the groups in a composite measure of health care utilization and medical complications, nor in psychosocial measures. Control participants reported fewer emergency/urgent care visits than treatment participants, thus one limitation of this study is that there may have been many participants who had little need for any intervention to address health or psychosocial outcomes. Perhaps results would be more robust if there had been identified participants with persistent problems requiring the intervention. Nevertheless, it appears that this direct to consumer intervention represents a promising approach to reducing health care utilization.

In another study [18], 137 participants with acute SCI from three sites in three different countries were randomized to teleSCI with video conference sessions, or to standard care. Treatment consisted of one teleSCI session every week for 2 months followed by nine teleSCI bimonthly sessions. Each session lasted $45 \mathrm{~min}$ and was one of two types; type one included a medical doctor and a nurse, and type two included a physiotherapist and/or an occupational therapist. Participants in the control group received the standard home-specialized, nursing-specialized, or unspecialized hospital care that they would normally have received after discharge. Outcomes included functional post-discharge status at 6 months, incidence of complications and participants' satisfaction with the healthcare services. The authors reported the mean between-group difference in their analyses for all the outcomes. Based on the findings of functional independence measure (FIM) total 
score (mean between group difference 4.31; 95\% CI 1.27-7.35) and FIM motor score (mean between group difference $4.31 ; 95 \%$ CI $1.24-7.38$ ) at one of the sites, the authors concluded that teleSCI direct to consumer may benefit patients discharged from a spinal cord unit, compared with standard care in terms of functional improvement. In a public health report [19], the effectiveness of teleSCI direct to consumer reduced the incidence of secondary conditions. 111 patients with SCI were randomized to video-conferencing using a compact equipment, telephone-using telephone line, or to standard care. Participants in the video and telephone groups received individualized educational sessions with a study nurse once a week for 5 weeks, then once every two weeks for 1 month in addition to standard care. Standard care provided participants a help line to call if and when they needed assistance prior to a 2-month post discharge visit. Interestingly, the authors did not report the mean between-group difference in outcomes in their analyses, but they concluded that telephone-or video-based interventions improve healthrelated outcomes for people with acute SCI living at home and that teleSCI may be cost-saving, using a direct to consumer strategy.

Emotional adjustment is an area that is amenable to treatment via telehealth. Dorstyn et al. [20] assessed the efficacy of telephone-based counseling vs. usual care to improve the emotional adjustment of 40 adults with SCI in Australia. All participants had recently been referred to inpatient psychology treatment, thus were considered to have baseline distress and in need of counseling post discharge. In the treatment group seven teleSCI sessions were delivered over a 12 -week period, by a single psychologist. Pre-intervention and post-intervention data, plus a 3-month follow-up assessment, were compared with that of an SCI control group who received standard care which consisted of a single face-to-face consultation with a psychologist at 3 months post discharge. TeleSCI participants reported clinical improvements in depression, anxiety and aspects of SCI coping immediately post-intervention. Delivery related outcomes, including participation rate and cost analyses, were positive; however not statistically significant. Effects were also minimal at the 3-month follow-up; thus lasting benefits of teleSCI were not demonstrated. Results suggest that "continued psychological services for individuals reporting distress during their inpatient rehabilitation are important, and can be delivered direct to consumer costeffectively, and efficiently by telephone [20]".

Other studies document the efficacy of teleSCI with regards to physical therapies. One group [21] in the US performed a pre-post study of a convenience sample of 16 chronic SCI individuals with shoulder pain and manual impingement on exam. A 12-week exercise program was coupled with therapist video monitoring for technique and exercise advancement. Outcomes including pain and function were measured at baseline, post intervention (12 weeks), and follow-up (>24 weeks) in a motion analysis laboratory. There was a significant main effect for pain and function between the three time points on the Wheelchair User's Shoulder Pain Index, Disabilities of Arm, Shoulder and Hand Index, and Shoulder Rating Questionnaire. Isometric strength measurements of the serratus anterior and scapular retractors significantly increased after the exercise intervention. Muscle impulse produced by the lower trapezius during a fatigue task also improved. Limitations of this study are that there was no control group with individuals being treated at a facility. Moreover, it would have been interesting to see a cost and satisfaction analysis. Another group [22] developed and examined the feasibility, reliability and validity of tele-assessment of balance and leg strength in 22 persons with chronic SCI. Motor and sensory scores showed significant improvements at 6 months; however, there were no changes in balance scores. Overall, this technique had excellent inter-rater reliability for measuring leg force at maximum flexion and at maximum extension, and excellent intra-rater reliability for measuring leg force at maximum flexion, thus documenting the ability to effectively assess SCI persons with regards to physical changes via teleSCI. Effectiveness of the individual-level impact of a previously piloted exercise counseling intervention [23-25] was evaluated among 65 community dwelling adults with SCI in Canada to promote leisure time physical activity (LTPA). Fifty three patients participated in the evaluation of a 6-month, individualized telephonecounseling program, with focus on developing and strengthening clients' social cognitions for engaging in selfmanaged LTPA. Efficacy evaluation showed that intentions for engaging in LTPA remained high throughout enrollment with a trend for more clients engaging in moderate-toheavy-intensity LTPA at 6 months $(52 \%)$ vs. baseline $(35 \%)(p=.09)$. Conclusions were that telephone-based counseling is a promising strategy for promoting community-based LTPA.

Another group [26] performed a pilot study of an oral home telecare program training in the use of assistive devices, using a rechargeable powered toothbrush with brushing time display, a flosser and a universal holder so that the oral hygiene device could be fastened to their hand, and PC-based videoconferencing between each participant and an occupational therapist. Training consisted of five 15-30 min sessions over 3 months. Gingival health assessment occurred at baseline, six and 12 months. Statistically significant differences in gingival inflammation and improved dental hygiene behaviors were reported at six and 12 months compared to baseline evaluation. Lack of high-speed internet services reduced the participation rate, as did lack of computer literacy and software 
incompatibilities. The authors commented on the inability to determine the extent of the contribution from the device vs. the telecare service.

Another utility of direct to patient teleSCI is for treatment and prevention of PUs. One group described a teleSCI system in Norway to provide specialist guided communitybased follow-up for patients with PUs [8]. Home-based follow-up was provided for seven patients with PUs using video-teleconferencing technology with PC-based videoconferencing equipment, and an external web-based camera in the patient's home. The pilot program was cost effective at $15 \%$ of the cost associated with a regular outpatient clinic appointment and $3 \%$ of the cost of an inpatient admission. Patients and home nursing services reported favorable experiences with this direct to consumer and provider to provider system. Concerns included funding and coding for such services. Further research and extension of services was recommended. In another pilot, randomized trial of 30 individuals, evaluation of teleSCI with the use of telephone contact as a model of community-based care for people with SCI in Bangladesh [27], outcomes were measured in formation of PUs, mortality, complications, depression, participation and quality of life. One participant in each group died, two participants in the control group, and three participants in the intervention group had PUs at 2 years. Three participants were hospitalized for serious PUs, and six indicated that PUs had been a moderate or severe problem in the preceding 3 months. In a newly published RCT [28] including 120 participants with PUs, using a telephonebased intervention, the authors found that PUs healed significantly better in the intervention group vs. the control group. Despite these results, the authors said that their results weren't conclusive for recommendations. However, taken together, these two trials $[27,28]$ demonstrate the feasibility of using teleSCI to provide regular follow-up direct to consumer, after discharge, for people with SCI in low-income and middle-income countries.

Hill et al. [29] did a validation study of home telehealth for pressure ulcer assessments among patients with SCI. They compared the reliability and validity of assessments made via telephone and videoconferencing with a reference method, and found that "the agreement on the presence of a PU was excellent for both telephone and videoconferencing approaches (92\% for telephone, 97\% for videoconferencing). The diagnoses of the stage of PU made via telephone and videoconferencing showed substantial to almost perfect agreement with the inperson diagnoses (Spearman's rho of 0.76 and 0.83 , respectively)". The authors write "There was a tendency for the measurements of wound volume to be somewhat larger in the telephone and videoconferencing modalities compared to those made in-person. Bland-Altman plots showed that videoconferencing gave substantially narrower $95 \%$ limits of agreement", and the authors concluded that "the findings indicate that telephone can be a useful tool for identifying the presence of a PU, but videoconferencing is required to obtain an evaluation reasonably close to that of a home visit [29]".

In some of the studies in the "direct to consumer" group, the teleSCI participants received additional support/ therapy, and this might have resulted in improved outcomes for the teleSCI group. Further research should compare telehealth with equal amonts of traditional therapy or therapy similar to that offered via telehealth. Then it will be possible to compare whether teleSCI is a more cost-effective and efficient way to deliver post discharge health services.

\section{Store and forward}

Only one study [30] was found showing good efficacy of using stored images of PUs for teleSCI. With regards to deciding a treatment plan, the total percentage agreement between the tele-assessment decision and the live decision strategy was good, with an inter-rater reliability of 0.96 (0.93-0.98) and an agreement with paper version of 0.90 (0.83-0.95), indicating that tele-assessment of PUs in individuals with SCI using digital images may be a feasible option.

\section{Web-based treatments}

Seven studies $[14,30-33,35,38]$ referred to online monitoring (Table 2), four of them considered primarily webbased treatments. [31-33, 35]. In a pilot study [31] of a web-based self-management system for people with SCI utilizing intermittent catheterization (IC), 30 persons with SCI were enrolled, and 22 completed the entire program, which included three phone calls with a continence nurse and an online forum. The study also tested new selfefficacy-scales and self-management scales at the beginning and end of the study; however, they found no statistically significant improvement. According to the authors, this study "indicates that a web-based education program, following inpatient rehabilitation and also years after IC training, particularly when supported by phone, nursing consultation and online discussion, is likely to be well received by people who have a SCI [31]".

In another article [32], the pre-treatment and posttreatment effect of an online self-help program, Psyfit was evaluated. Seven out of 14 participants with SCI completed the modules, and the authors reported an increase in mental health, but a non-significant change in well-being. It was proposed that Psyfit may be a feasible program, but there were no significant results to support this in the article. The authors recommended further controlled studies, and they also recommended the use of teleSCI as a diagnostic tool. 


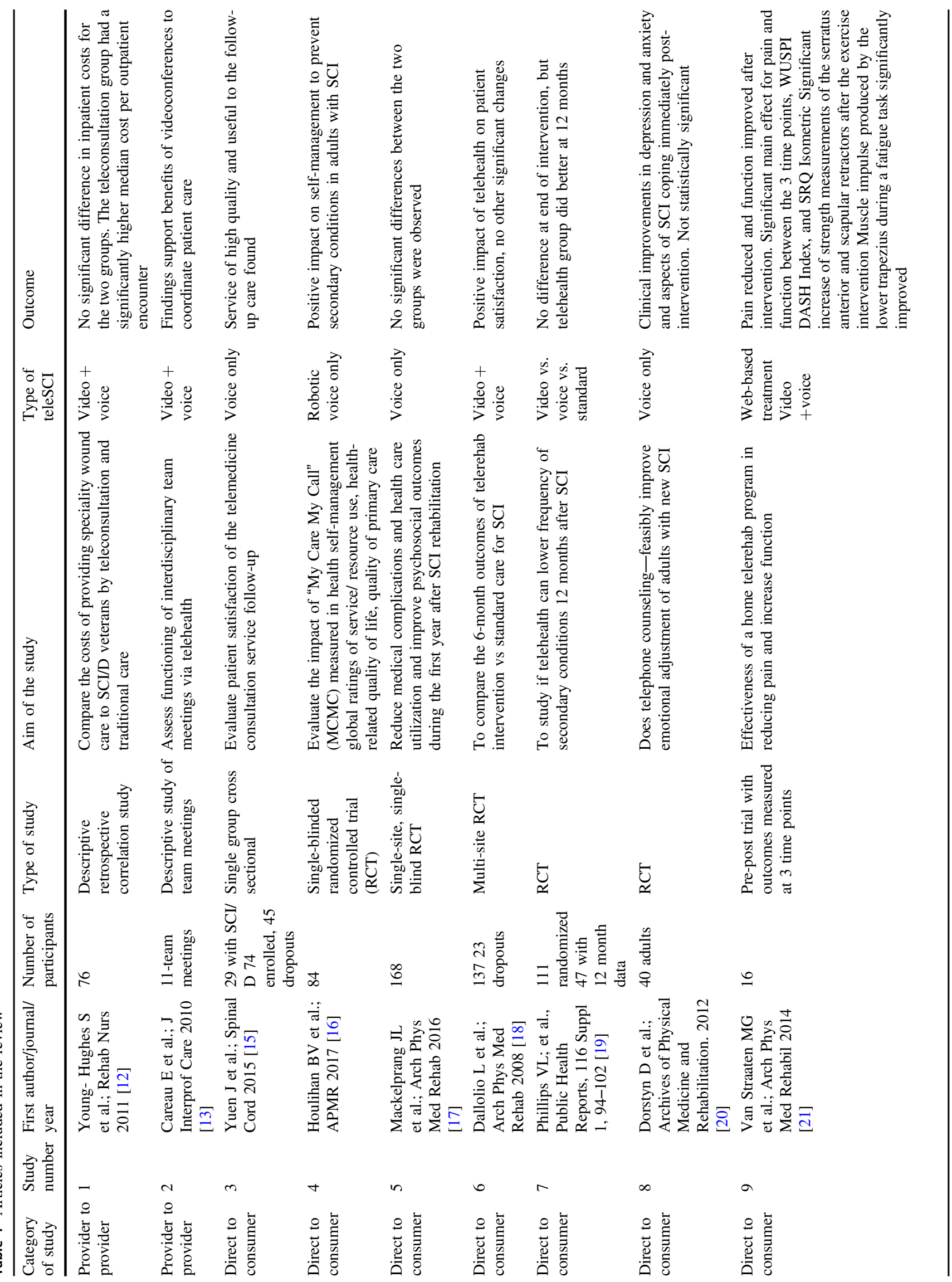




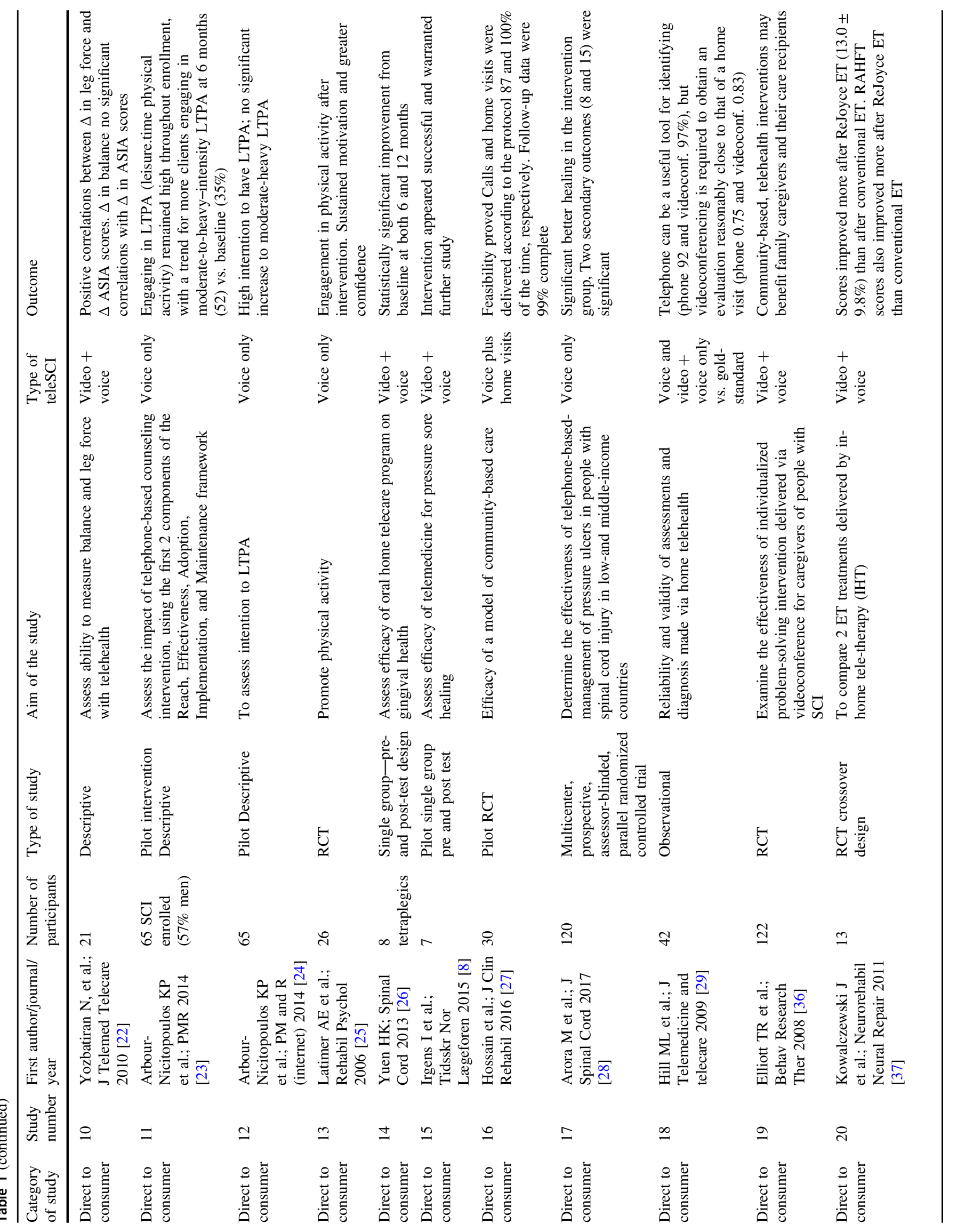




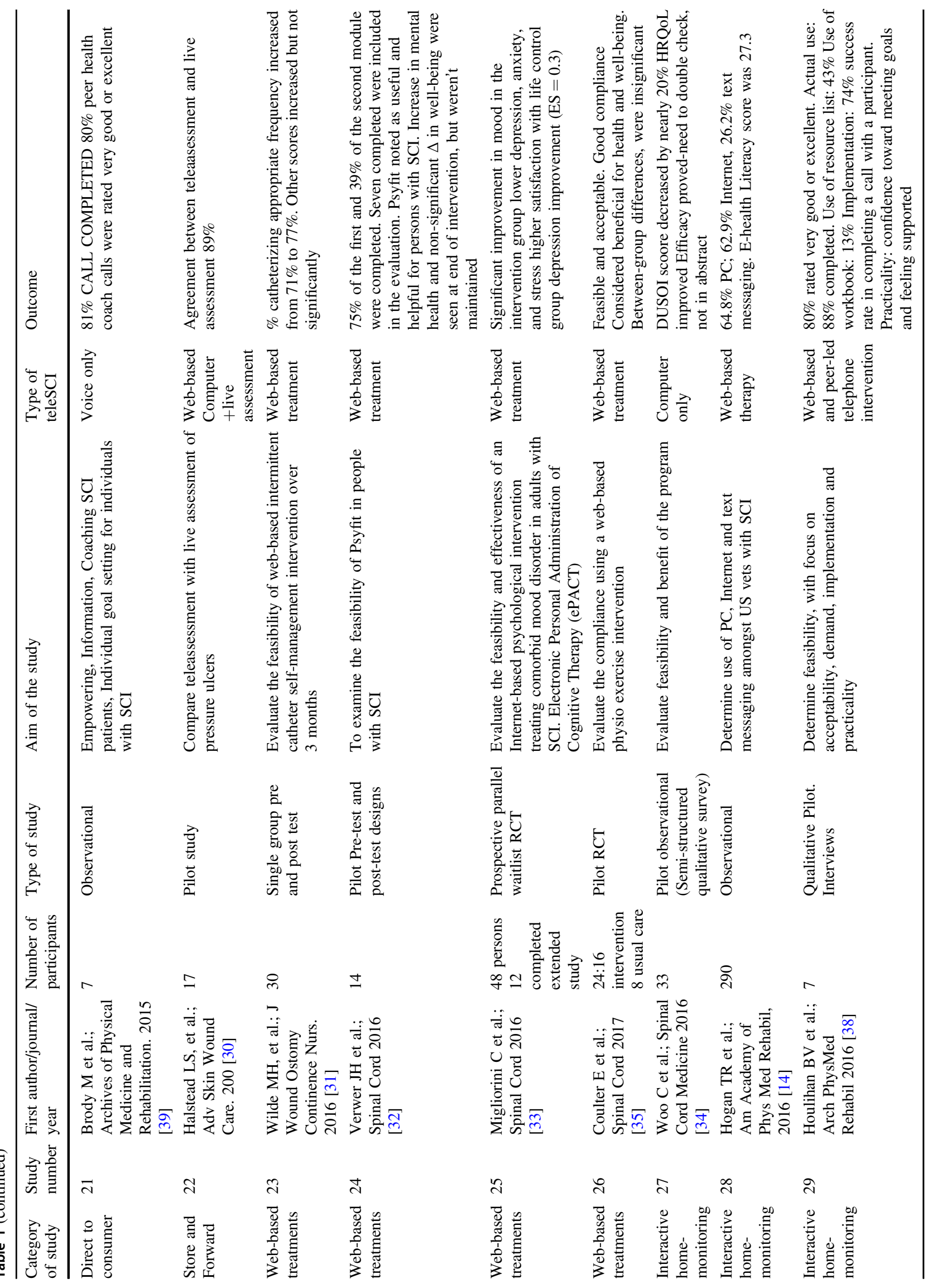


Table 2 Characteristics of studies included in the review

\begin{tabular}{lllll}
\hline Category of the study & \multicolumn{3}{l}{ Modalities of teleSCI } \\
\cline { 2 - 5 } & Voice only & Video plus voice & Data only & Robotic voice or video \\
\hline Provider to provider & 11 & 2 & 1 & \\
Direct to consumer & & & 1 & 2 \\
Store and Forward & 1 & 3 & \\
Web-based treatments & 1 & 2 & \\
Interactive Home Monitoring & 1 & & & \\
\hline
\end{tabular}

Some of the studies utilized more than one modality

\section{Interactive home monitoring}

A descriptive report [34] discussed home monitoring with data messaging devices in $33 \mathrm{SCI}$ males. The device included about 400 content items assessing a broad range of symptoms, educational needs, and self-management behavior recommendations. Improvement in clinical outcomes, care management practices, clinical flow processes and issues that needed to be addressed prior to broader protocol implementation were described in a subset of patients. Issues included accessibility and confidentiality concerns related to the program; however, overall the program was touted as a potentially excellent self-management, early education, home-based tool for newly injured SCI persons. Increased access to specialty care and early information received via the devices was hypothesized as potentially beneficial in allowing for earlier interventions and prevention of symptom worsening.

\section{Discussion}

In this review we have studied the status of to whom and how teleSCI has been offered for the last 20 years.

The actual number of articles included in the review was 29. Twelve of the studies were conducted as RCTs, nine were pilot studies, and the remainder were conducted as non-randomized, observational, or descriptive studies, mainly to see if teleSCI interventions were feasible (Table 1). Different aims and outcomes, and lack of information about selection criteria, different categories, and modalities, are weaknesses in the studies. As commented by Wilde et al. [31], "because of the small the number of participants, small changes could not reach significance, even if there was a trend". These small sample sizes, makes it difficult to make any unified recommendations for the whole SCI population [31] or for further development.

Even though there are indications that teleSCI can be an effective means to communicate and collaborate, we are not yet able to conclusively document that teleSCI is an optimal means of interacting between health care professionals or between professionals and people with SCI/D.
Additionally, as this is an evolving field, the safety and security concerns needs further research.

Overall, studies highlight current issues in the performance of teleSCI. Yuen et al. [15], discuss the lack of high speed internet services reducing participation rate as did lack of computer literacy and incompatibility of participants' computers with the videoconferencing program. The need for caregiver assistance to use an at-home-monitoringprogram was emphasized by Woo et al. [34]. However, with successful provision of services there was high patient satisfaction [26] and services were found to be cost effective [8].

A confounding factor may be that a number of the studies $[8,17]$ didn't evaluate the necessity of participating in the study. Thus, they may have included individuals with little need for interventions to address health or psychosocial outcomes, and findings may not be as robust as they would have been if all participants had clinical needs. Additionally, some studies could be placed in multiple categories (Tables 1,2), making between study comparisons difficult. Other studies [21] compared various treatments performed via teleSCI, thus they were actually not assessing teleSCI vs. standard care. This makes it difficult to compare the use of teleSCI with other interventions, as well as to compare the potential of teleSCI to prevent development of complications. Therefore there are still unsolved questions about whether teleSCI is a good thing for people with SCI/D, and if it is good, for what acute and chronic problems is it better than in person care, for which is it inferior to in person care, and for which ones is it similar to face- to- face? We didn't manage to find conclusive answers on financial implications or if there are adverse events to be aware of. We recommend to bear this in mind when future trials are planned.

\section{Future recommendations for teleSCI}

There are many companies developing telemedicine services to provide routine care to able-bodied individuals, and many benefits of telehealth are being recognized. Telehealth is environmental friendly with a lower impact in $\mathrm{CO} 2$ emissions, and lower cost due to a decrease in travel. 
Telehealth may also be ideal to use in times of disaster. It is convenient and saves time for the patient and high patient satisfaction rates have been found. Health care services like telepsychology [35] are relatively commonplace and telemedicine used in the group of patients suffering sequelae after stroke [6] is routinely used in rural hospitals. Moreover, insurance carriers are realizing the benefits of telehealth and it is being frequently reimbursed. Telemedicine is also being used more and more for emergency care and crisis management [7]. The lack of qualified providers, the high costs and burden of transportation for persons with SCI and their frequent common acute health problems, such as urinary tract infections, pressure sores and respiratory concerns, make teleSCI an ideal way to provide care. Similarly, long-term concerns, such as spasticity and pain management, are amenable to intermittent visits via teleSCI rather than repetitive visits to the clinic. Moreover, similar to the able-bodied population, the provision of psychotherapy, physical, occupational, speech and vocational services to persons with SCI/D, via teleSCI is appropriate. Finally, teleSCI to rural hospitals by an SCI specialist, could bring the possibility for more accurate diagnosis of acute SCI, so that optimal therapy could be provided.

As can be seen from this report, the predominant format that has been tested to date regarding teleSCI involves delivery of care directly from the provider to patient's homes. Store and forward is routinely used in radiology; however, limited research has been done on its benefits in the SCI population. Provider to provider would be an excellent platform for collaboration, and to develop services for persons with SCI. This is because the number of specialized professional caring for persons with SCI is limited; however, these services have not been tested. Moreover, provider to patient may also be performed in this type of setting when a provider at one facility provides care to a patient at a remote facility. Web-based treatments where the consumer receives therapy or training via a program accessed via the internet has begun to be developed for SCI, but such services are still in their infancy. Interactive home monitoring or the provision of automated monitoring services to patients in their homes, are beginning to be developed.

Developing a teleSCI program requires resolution of defined key issues to ensure services are safely provided. Use of a secure communication platform is necessary to ensure appropriate confidentiality of patient information. Appropriate consent must be provided, and safe and secure downloading of the necessary software must be given priority. State and country licensure regulations for services provided must be considered as must privilege concerns and accreditation processes. As regulations for these services are varied, it is important for the provider to be knowledgeable about the exact locations they are practicing.
One major barrier for the provision of teleSCI services is the issue of insurance. Local and national issues need to be taken into account. Finally, the issue of reimbursement must be investigated. Although some national and private insurance companies are providing reimbursement for telehealth, the rules are constantly changing and each system and provider will need to determine the cost/benefit of the provision of teleSCI services. Additionally, equipment needs and whether peripheral devices are necessary to view details of PUs must be considered.

TeleSCI has the possibilities to support both patients and providers with face-to-face communication at a distance. It seems to be a useful, safe and cost-effective tool applied in different modalities, by different members of the multi-and interdisciplinary team, with possibilities for better user participation from the patients. Future work will require standards of care for teleSCI services and research that addresses user-participation, safety, efficacy, cost-benefit and quality of life. A standardized dataset may also be appropriate to describe telehealth interventions for persons with SCI. This should allow future research to be conducted in a fashion that will allow comparison of patient populations, accessibility to technology, treatment interventions and efficacy.

\section{Conclusion}

The use of telemedicine has expanded rapidly [1], and both inpatient and outpatient services are available directly both to patient and caregivers for common concerns. These services seem to be socioeconomically and environmentally favorable. Yet, we do not have enough evidence about optimal methods for utilization, methodology and efficacy of teleSCI. We need continued research to evaluate, so that further development and expansion will be for the best of both caretakers and caregivers.

Acknowledgements The authors would like to thank Senior Librarian, Gunn Kleven, at the Oslo University Library for help, advice and guidance with the literature searches. Also thanks to colleagues, Phd. Solveig Lægreid Hauger and Professor Magne Thoresen for the input and advice during the review process

Author contributions All the authors contributed in designing the review protocol. The first author (II) conducted the initial search together with the librarian, and the last author (MAl) double-checked the selection. II and TR, NL and FB-S, MAr and RM, extracted the data. Any disagreement was resolved by MAl II and MAl prepared the figures and wrote the manuscript. All authors edited and critical reviewed the manuscript, and approved the final version of the manuscript.

Funding II is founded by the Norwegian ExtraFoundation for health and rehabilitation (Grant 2017/FO149043). The founding partner has no involvement in the methodological aspect of the project. 


\section{Compliance with ethical standards}

Conflict of interest The authors declare that they have no conflict of interest.

Open Access This article is licensed under a Creative Commons Attribution-NonCommercial-NoDerivatives 4.0 International License, which permits any non-commercial use, sharing, distribution and reproduction in any medium or format, as long as you give appropriate credit to the original author(s) and the source, and provide a link to the Creative Commons license. You do not have permission under this license to share adapted material derived from this article or parts of it. The images or other third party material in this article are included in the article's Creative Commons license, unless indicated otherwise in a credit line to the material. If material is not included in the article's Creative Commons license and your intended use is not permitted by statutory regulation or exceeds the permitted use, you will need to obtain permission directly from the copyright holder. To view a copy of this license, http://creativecommons.org/licenses/by-nc-nd/4.0/.

\section{References}

1. Center for connected Health Policy. What is Telehealth? 2017. http://www.cchpca.org/what-is-telehealth.

2. National Center for Interaction and Telemedicine. Advanced informatics in Medicine. 1991. www.telemed.no/omNST/.

3. Thrall, J.H. Teleradiology: part I history and clinical applications. Radiology 2007;243(3):613-7.

4. Trnka P, White M, Renton W, McTaggart S, Burke J, Smith A. A retrospective review of telehealth services for children referred to a paediatric nephrologist. BMC Nephrol 2015;16:12.

5. Jani PD, Forbes L, Choudhury A, Preisser J, Vieira AJ, Garg S. Evaluation of diabetic retinal screening and factors for ophthalmology referral in a telemedicine network. JAMA Ophthalmol. 2017. https:// doi.org/10.1001/jamaophthalmol.2017.1150. Epub ahead of print.

6. Wechsler LR, Demaerschalk BM, Schwamm LH, Adeoye OM, Audebert HJ, Fanale CV. American heart association stroke council; council on epidemiology and prevention; council on quality of care and outcomes research. telemedicine quality and outcomes in stroke: a scientific statement for healthcare professionals from the american heart association/american stroke association. Stroke 2017;48(1):e3-25. https://doi.org/10.1161/ STR.0000000000000114.

7. Thomas JT, Moeckli J, Mengeling MA, Goedken CC, Bunch J, Cram P, et al. Bedside critical care staff use of intensive care unit telemedicine: comparisons by intensive care unitcomplexity. Telemed J E-Health. 2017. https://doi.org/10.1089/tmj.2016.0243. Epub ahead of print.

8. Irgens I, Kleven L, Sorli H, Stanghelle JK, Rekand T. Telemedicine brings specialist healthcare services to patients' homes. Tidsskrift for Den Norske Legeforening 2015;135(19):1716-7. https://doi.org/10.4045/tidsskr.15.0770

9. Alexander M, Irgens I, Marshall R, Houlihan B, Arora M, Liu N, et al. Around the world with TeleSCI. ISCoS 2016; Workshop 16: Abstract no $208 \mathrm{http}: / / w w w . i s c o s . o r g . u k / u p l o a d s / A B S T R A C T S /$ ISCoS 2016_ABSTRACT_BOOK.pdf

10. Ferrari M, Writing narrative style literature reviews. Eur Med Writ Assoc. 2015;24(4):230-5.. https://doi.org/10.1179/2047480615Z. 000000000329 .

11. Green BN, Johnson CD, Adams A. Writing narrative literature reviews for peer-reviewed journals: secrets of the trade. J Chiropr Med 2006;5(3):101-17.

12. Young-Hughes S. Spinal Cord injury/disorder teleconsultation outcome study. Rehabil Nurs. 2011;36:153-72.
13. Careau E, Dussault J, Vincent C. Development of interprofessional care plans for spinal cord injury clients through videoconferencing. J Interprof Care 2010;24(1):115-8.

14. Hogan TP, Hill JN, Locatelli SM, Weaver FM, Thomas FP, Nazi KM. et al.Health information seeking and technology use among veterans with spinal cord injuries and disorders.Am Acad Phys Med Rehabil. 2016;8:123-30.

15. Yuen J, Thiyagarajan CA, Belci M. Patient experience survey in telemedicine for spinal cord injury patients. Spinal Cord 2015;53 (4):320-3. http://search.ebscohost.com/login.aspx?direct=true $\& \mathrm{db}=$ cin $20 \& \mathrm{AN}=103786785 \&$ site $=$ ehost-live

16. Houlihan BV, Brody M, Everhart-Skeels S, Pernigotti D, Burnett $\mathrm{S}$, Zazula J, et al. Randomized trial of a peer-led, telephone-based empowerment intervention for persons with chronic spinal cord injury improves health self-management. Arch Phys Med Rehabil 2017;98:1067-76.

17. Mackelprang JL. Outcomes and lessons learned from a randomized controlled trial to reduce health care utilization during the first year after spinal cord injury rehabilitation: telephone counseling versus usual care. Arch Phys Med Rehabil 2016;97:1793-6.

18. Dallolio L, Menarini M, China S, Ventura M, Stainthorpe A, Soopramanien A, et al. Functional and clinical outcomes of telemedicine in patients with spinal cord injury. Arch Phys Med Rehabil 2008;89(12):2332-41.

19. Phillips VL, Vesmarovich S, Hauber R, Wiggers E, Egner A. Telehealth: reaching out to newly injured spinal cord patients. Pub Health Rep 2001;116 Suppl 1:94-102.

20. Dorstyn D, Mathias J, Denson L, Robertson M. Effectiveness of telephone counseling in managing psychological outcomes after spinal cord injury: a preliminary study. Arch Phys Med Rehabil 2012;93(11):2100-8.

21. Van Straaten MG, Cloud BA, Morrow MM, Ludewig PM, Zhao KDVan, Effectiveness of home exercise on pain, function, and strength of manual wheelchair users with spinal cord injury: a high-dose shoulder program with telerehabilitation. Arch Phys Med Rehabil. 2014;95(10):1810-7

22. Yozbatiran N, Harness ET, Le V, Luu D, Lopes CV, Cramer SC. A tele-assessment system for monitoring treatment effects in subjects with spinal cord injury. J Telemed Telecare 2010;16 (3): $152-7$.

23. Arbour-Nicitopoulos KP, Tomasone JR, Latimer-Cheung AE, Martin Ginis KA. Get in motion: an evaluation of the reach and effectiveness of a physical activity telephone counseling service for canadians living with spinalcord injury. Phys Med Rehabil. 2014;6(12):1088-96.

24. Arbour-Nicitopoulos KP, Ginis KA, Latimer AE. Planning, leisure-time physical activity, and coping self-efficacy in persons with spinal cord injury: a randomized controlled trial. Arch Phys Med Rehabil 2009;90:2003-11.

25. Latimer AE, Ginis KAM, Arbour KP. The efficacy of an implementation intention intervention for promoting physical activity among individuals with spinal cord injury: a randomized controlled trial. Rehabil Psychol 2006;51:273-80.

26. Yuen HK. Effect of a home telecare program on oral health among adults with tetraplegia: a pilot study. Spinal Cord 2013;51 (6):477-81. https://doi.org/10.1038/sc.2012.176

27. Hossain MS, Harvey LA, Rahman MA, Bowden JL, Islam MS, Taylor V, et al. A pilot randomised trial of community-based care following discharge from hospital with a recent spinal cord injury in Bangladesh. J Clin Rehabil 2016;1-9.

28. Arora M, Harvey LA, Glinsky JV, Chhabra HS, Hossain S, Arumugam N, et al. Telephone-based management of pressure ulcers in people with spinal cord injury in low- and middleincome countries: a randomized controlled trial. Spinal Cord. 2017;55:141-7. 
29. Hill ML, Cronkite RC, Ota DT, Yao EC. Validation of home telehealth for pressure ulcer assessment: a study in patients with spinal cord injury. J Telemed Telecare. 2009;15:196-202.

30. Halstead LS, Dang T, Elrod M, Convit RJ, Rosen MJ, Woods S. Teleassessment compared with live assessment of pressure ulcers in a wound clinic: a pilot study. Adv Skin Wound Care. 2003;16:91-6.

31. Wilde MH, McMahon JM, Fairbanks E, Brasch J, Parshall R, Zhang F. Feasibility of a web-based self-management intervention for intermittent urinary catheter users with spinal cord injury. J Wound Ostomy Cont Nurs. 2016;43:529-38. https://doi.org/10. 1097/WON.0000000000000256

32. Verwer JH, van Leeuwen CM, Bolier L, Post MW. Feasibility of an online well-being intervention for people with spinal cord injury: a pilot study. Spinal Cord. 2016;54:473-7.

33. Migliorini C, Sinclair A, Brown D, Tonge B, New P. A randomised control trial of an Internet-based cognitive behaviour treatment for mood disorder in adults with chronic spinal cord injury. Spinal Cord. 2016;54:695-701.

34. Woo C, Seton JM, Washington M, Tomlinson SC, Phrasavath D, Farrell KR, et al. Increasing specialty care access through use of an innovative home telehealth-based spinal cord injury disease management protocolSCI DMP) J Spinal Cord Med 2014.
35. Coulter EH, McLean AN, Hasler JP, Allan DB, McFadyen A, Paul I. The effectiveness and satisfaction of web- based physiotherapy in people with spinal cord injury: a pilot randomized controlled trial. Spinal Cord. 2017;55:383-9.

36. Elliott TR, Brossart D, Berry JW, Fine PR. Problem-solving training via videoconferencing for family caregivers of persons with spinal cord injuries: a randomized controlled trial. Behav Res Ther. 2008;2008(46):1220-9.

37. Kowalczewski J, Chong SL, Galea M, Prochazka A. In-home telerehabilitation improves tetraplegic hand function. Neurorehabil Neural Repair. 2011;25:412-22.

38. Houlihan BV, Everhart-Skeels S, Gutnick D, Pernigotti D, Zazula J, Brody M, et al. Empowering adults with chronic spinal cord injury to prevent secondary conditions. Arch Phys Med Rehabil. 2016;97(1687-95):e5 https://doi.org/10.1016/j.apmr. 2016.04.005

39. Brody M, Houlihan BV, Skeels SE, Zazula J, Pernigotti D, Mercier HW, et al. Development of a peer-led phone intervention for goal-setting health care needs in spinal cord injury. Arch Phys Med Rehabil. 2015;96:e19 http://ovidsp.ovid. com/ovidweb.cgi? $\mathrm{T}=\mathrm{JS} \& \mathrm{CSC}=\mathrm{Y} \& \mathrm{NEWS}=\mathrm{N} \& \mathrm{PAGE}=$ fulltext $\& \mathrm{D}=$ emed17\&AN $=72088523$. 